\title{
ISDN 1-56072-054-8
}

